\title{
Singapore Zebrafish
}

National Cancer Institute

\section{Source}

National Cancer Institute. Singapore Zebrafish. NCI Thesaurus. Code C79980.

A wild-type zebrafish line, the stock of which was obtained from a fish dealer in Singapore. 